Nonlinear Processes in Geophysics, 12, 451-460, 2005

SRef-ID: $1607-7946 / \mathrm{npg} / 2005-12-451$

European Geosciences Union

(C) 2005 Author(s). This work is licensed

under a Creative Commons License.

\title{
Continuous partial trends and low-frequency oscillations of time series
}

\author{
A. R. Tomé ${ }^{1}$ and P. M. A. Miranda ${ }^{2}$ \\ ${ }^{1}$ Universidade da Beira Interior, Department of Physics, and Centro de Geofísica da Universidade de Lisboa, Portugal \\ ${ }^{2}$ University of Lisbon, Faculdade de Ciências, Centro de Geofísica, Portugal
}

Received: 15 June 2004 - Revised: 8 December 2004 - Accepted: 7 April 2005 - Published: 21 April 2005

Part of Special Issue "Nonlinear analysis of multivariate geoscientific data - advanced methods, theory and application"

\begin{abstract}
This paper presents a recent methodology developed for the analysis of the slow evolution of geophysical time series. The method is based on least-squares fitting of continuous line segments to the data, subject to flexible conditions, and is able to objectively locate the times of significant change in the series tendencies. The time distribution of these breakpoints may be an important set of parameters for the analysis of the long term evolution of some geophysical data, simplifying the intercomparison between datasets and offering a new way for the analysis of time varying spatially distributed data. Several application examples, using data that is important in the context of global warming studies, are presented and briefly discussed.
\end{abstract}

\section{Introduction}

The linear trend constitutes the most straightforward assessment of the long-term behavior of a time series. However, real time series are generally not well fitted by a straight line and the use of other analytical functions always raises difficult interpretation problems. In practice, many data analysis start by a subjective inspection of the time series graphic often revealing important features of the data, such as periodicities, trends, localized anomalies, localized changes in the trend or in other statistics that may contribute to an understanding of the underlying physics. Linear analysis, such as linear trend fitting or Fourier analysis, cannot deal with heterogeneity in the data, and are blind to many of the features that may be present.

The detection of heterogeneity is central in many geophysical problems, namely in the analysis of Global Change. Some methods, such as DFA (Detrended Fluctuation Analysis, Peng et al., 1994) divide the times series in fixed inter-

Correspondence to: A. R. Tomé (atome@dfisica.ubi.pt) vals and perform a best fit, in the least squares sense, in each of the subintervals. By doing so, those methods focus on local aspects of the time series behavior, emphasizing heterogeneity. However, the fitting functions present, in most cases, strong discontinuities at the interval boundaries and the method doesn't offer a global description of the data.

Fourier methods deal well with oscillations in the data and always produce a global analysis. However, those methods impose periodicity of the time series and don't cope well with discontinuities in the data. One may use powerful spectral methods to analyze the local behavior of time series in moving time windows and assess the slow change in the spectra, as done in the Multi Singular Spectral Analysis (Vautard and Ghil, 1989; Vautard et al., 1992). The analysis is, though, rather complex and doesn't deal with the simplest problems of the change in trend.

Here, we propose a simple non-linear approach that mimics the subjective analysis of a time series graphic that one may perform with a pen on a piece of paper, yet using an objective numerical method that minimizes the mean square error of the fitting. The method consists in fitting the data with a set of continuous line segments, where the number of segments, the location of the breakpoints between segments, and the slopes of the different segments are simultaneously optimized. Only the overall fit is non-linear as the result is made of linear segments. The method was originally motivated by the study of Karl et al. (2000) on the changing trend of global warming and constitutes an extension of the method proposed by Tomé and Miranda (2004).

Section 2 describes the method, Sect. 3 presents some examples of application, Sect. 4 shows that this univariate method may be useful in the analysis of spatially distributed climate data, Sect. 5 addresses the problem of the statistical significance of the computed partial trends in the presence of noise. 


\section{The method}

Let us consider a generic time series of $n$ elements,

$\left\{y\left(t_{i}\right)\right\} \quad i=1, \ldots, n$

One wants to fit, in a least-squares sense, an unknown number of continuous line segments to it. Obviously, connecting all the data points by a strait line leads to the best, but also the trivial and useless solution with a null square residual sum. There need to be some additional conditions imposed to the solutions. The two most obvious possible conditions are to limit the number or the length of the line segments. Let us start with the first possibility. Let $m+1$ be the number of line segments, $m$ is therefore the number of data points where two different line segments of the solution meet. Following the terminology of Tomé and Miranda (2004) we call it breakpoints. The idea is then to perform the best fit of the piecewise linear function

$$
y\left(t_{i}\right)=c+\sum_{k=0}^{m m}\left(b_{k}-b_{k+1}\right) T_{k}+b_{m m+1} t_{i}
$$

$m m$ is chosen such that: $m m=0, \ldots, m$ and the $t_{i}$ data point belongs to $T_{m m} \leq t_{i} \leq T_{m m+1}$ of the time series (Eq. 1). The first, and probably the most important, unknown is the value of $m$. After knowing $m$, this function (Eq. 2) has $2 m+2$ unknowns: the $m$ time positions of the breakpoints $T_{m m}$, $m m=1, \ldots, m$, the $m+1$ slopes of the line segments $b_{k}$, $k=1, \ldots, m+1$ and the fitting function value at the origin, $c$. For this concise writing of the fitting function we take $T_{0}=0$ and $T_{m+1}=t_{n}$. This non linear fitting can be performed using, among many others possibilities, the Tensolve package, a software package for solving systems of nonlinear equations and nonlinear least squares problems using tensor methods by Bouaricha and Schnabel (1997) and freely available at http://www.netlib.org/toms/768. Nevertheless, as usual in non linear algorithms, an initial solution is required and the convergence process is strongly dependent on that first guess. An inadequate first guess could lead to an erroneous final solution. The authors have applied this methodology to some temperature time series of Portuguese station data using as initial solution the results of Karl et al. (2000) for the global mean temperature, with satisfactory results.

The need of a suitable first guess implies that an a priori study of each time series must be done before computing the fitting. However, regardless of the approach followed, every solution of a non linear system of equations is obtained by iteratively solving systems of linear equations, until some convergence criterion is attained. So, instead of using a generic non linear problem solver one can find a more suitable set of linear systems to solve, in order to obtain the best possible solution of the non linear problem.

The non linear function (Eq. 2) becomes a linear function if one imposes the values of the $m$ breakpoint positions, $T_{m m}$, $m m=1, \ldots, m$. In that case the fitting problem could be put as a linear over-determined system of equations of the type:

$\min \|\boldsymbol{y}-\mathbf{A} \boldsymbol{s}\|$, where $s$ is one ranking matrix solution of $m+2$ elements, $\boldsymbol{s}=\left[b_{1}, b_{2}, \ldots, b_{m}, b_{m+1}, c\right]$ and $\mathbf{A}$ is a constant coefficient $[n \times m+2]$ matrix. The algorithm to build the $\mathbf{A}$ matrix is given by Tomé and Miranda (2004).

The solution of the non linear system can then be obtained by solving the linear problem (Eq. 3) for all possible values of the breakpoint positions and, at the end, by choosing the solution that minimizes the residual square sum. In this way, one does not need to establish a convergence criterion. The price to pay for the simplicity of the approach is the fact that the number of linear systems to solve increases exponentially as the number $n$ of time series elements and the number $m$ of breakpoints increase, making the methodology unusable for long series with many possible breakpoints. In this case, the non-linear solver is also very expensive.

Fortunately, many relevant problems of low frequency variability or change in geophysics imply a moderate number of breakpoints and may be described by a not too large number of data points. By limiting the number of breakpoints and the minimum distance between consecutive breakpoints, one may reduce very substantially the number of times the system (Eq. 3) has to be solved. That is the case, for example, of global warming, which is well represented by annual mean temperature and for which one is interested in oscillations or trends at the interdecadal or longer time scales. In those cases, the computational constraints turn out to be irrelevant.

To implement the proposed method one must decide the number of breakpoints $m$. Clearly, without additional conditions, the residual square sum diminishes as the number $m$ increases and so it cannot be used to compute $m$. Karl et al. (2000) used Haar Wavelets to locate discontinuities in the mean global temperature series leading directly to the linear system (Eq. 3). Alternatively, we propose a rather simple approach, which has the advantage of giving a clear meaning to the breakpoints, and consists in selecting the set of $m$ breakpoints that best fits the data, in the least squares sense, and satisfies two simultaneous conditions: a minimum time distance between breakpoints and a minimum trend change at each breakpoint. In terms of the algorithm, this latter condition does not require any special process, but only the rejection of the solutions of the equations system (Eq. 3) that do not lead to a minimum change of the trend at the breakpoint.

The minimum interval between two consecutive breakpoints is the essential free parameter of the method. This interval, which is the minimum time window of the trend analysis, acts as a low-pass filter. The size of the window is optional, but, in principle, it is desirable that most of the breakpoint solutions stay apart by a value larger than the window size. If not, the window size is probably too small and one should be careful in interpreting the breakpoint distribution as reflecting the low frequency behavior of the time series.

Another important issue in partial trend analysis is the occurrence of end effects in the fitting process (Soon et al., 2004; Mann, 2004). In the proposed methodology it is straightforward to relax the condition on the minimum distance between consecutive breakpoints at the series bound- 


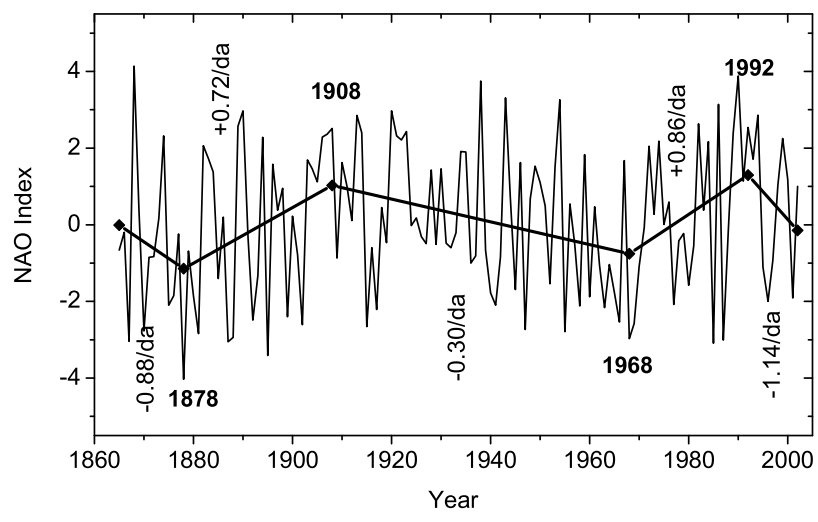

Fig. 1. Continuous linear trends for the NAO index. The minimum amount of change at breakpoints is $0.1 /$ decade. 20 years is the minimum allowed interval between breakpoints, and 5 years the minimum length allowed for the first and last segment.

aries. By doing so, one allows the method to adjust smaller linear segments at both ends, avoiding significant artificial constrains on the adjacent segments. The small end segments are, though, poorly constrained.

\section{Application examples}

\subsection{The North Atlantic Oscillation Index}

During the last decade the North Atlantic Oscillation Index (NAO) has been the subject of several studies (e.g. Hurrell, 1995; Jones et al., 1997; Ostermeier and Wallace, 2003), some of which evaluated overall trends, trends for limited periods and decadal trends. The significant correlation of the NAO index with precipitation in some regions of Europe and the existence of low frequency variability in the index have made it a target for many studies aiming to either explore its potential for seasonal forecasting or to understand recent climate change.

Figure 1 presents the NAO index from 1865 till 2002, together with the linear fit and the continuous line segments obtained by the proposed method. The latter were obtained by imposing a minimum change at breakpoints of $0.1 /$ decade in the NAO index partial trend, and a minimum trend duration of 20 years, except at the end segments where the minimum was put at 5 years. Figure 2 shows different fitting solutions, for different values of minimum length of the individual segments, always imposing a minimum of 5 years for the length of the end segments. When one allows segments with 10 years or more, the NAO time series is best approximated by segments near the shortest accepted length, with a change in trend sign at all breakpoints, except in the period between 1968 and 1992 where the index revealed a sustained positive trend. Those breakpoints in the series evolution seem robust as they are detected by the method in all solutions with minimum segment lengths up to 25 years. Above that value the imposed condition on the segment length is incompatible

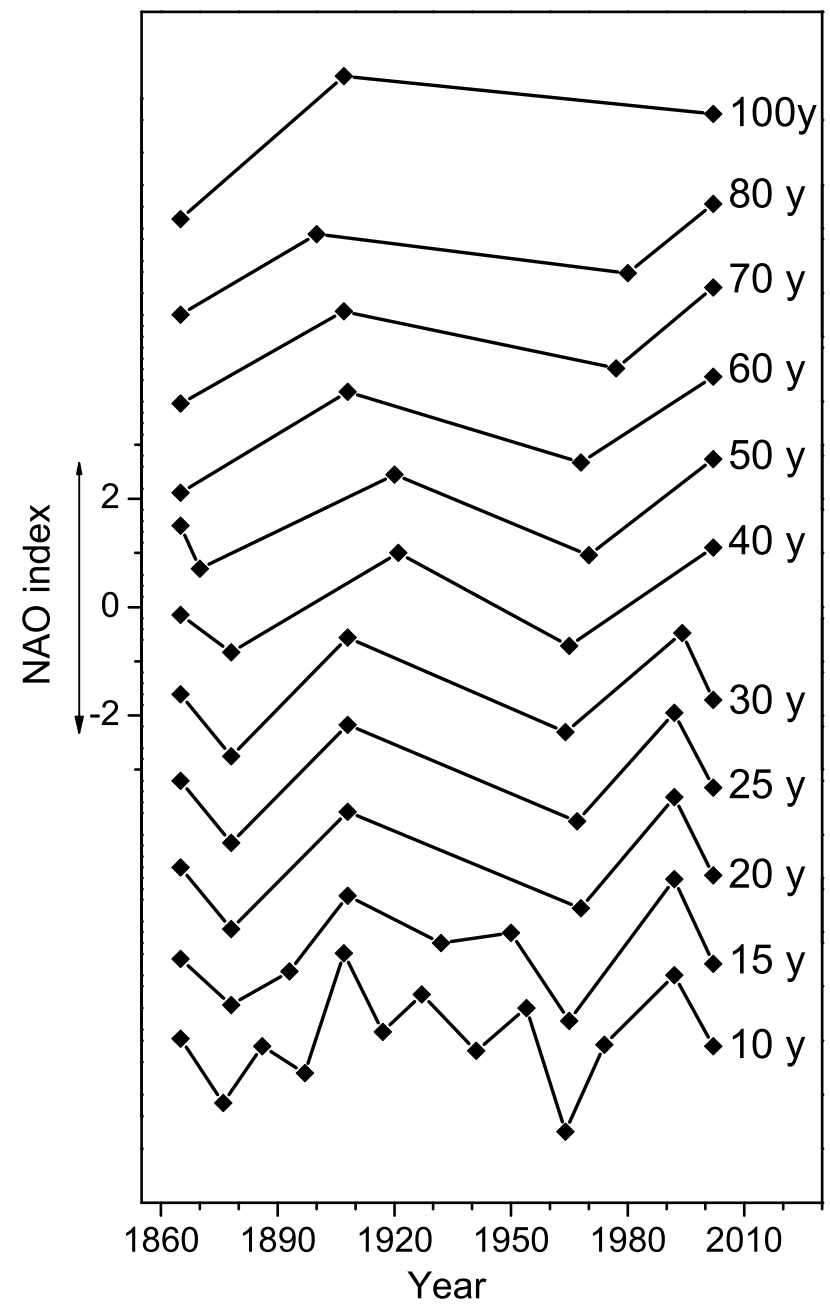

Fig. 2. Fitting of continuous linear segments to the NAO index, for different choices of the minimum trend duration.

with the location of those two breakpoints. The anomalous behavior of the index in the period 1968 to 1992, already noticed by Ostermeier and Wallace (2003) using a completely different method, is the main conclusion of this analysis. On the other hand, the recent downward trend of NAO in the last dozen years is an important fact to keep in mind when analyzing the recent evolution of climate in the North Atlantic sector, as shown below. It is also worth mentioning that the fastest rate of NAO evolution, excluding the shorter end periods, happens in the period 1968 till 1992.

The strong overall increase of the NAO index from 1968 until 1992 has been one of the reasons that justified the prominent role of this index in recent discussions of global warming. However, unlike mean temperature, which in principle can increase continuously, the NAO index is constrained by atmospheric mass conservation and so it is bound to oscillate around some mean value, whether its variation is internally generated or anthropogenicaly forced.

In spite of some agreement among several authors on the "anomalous" behavior of NAO index in the last decades, 


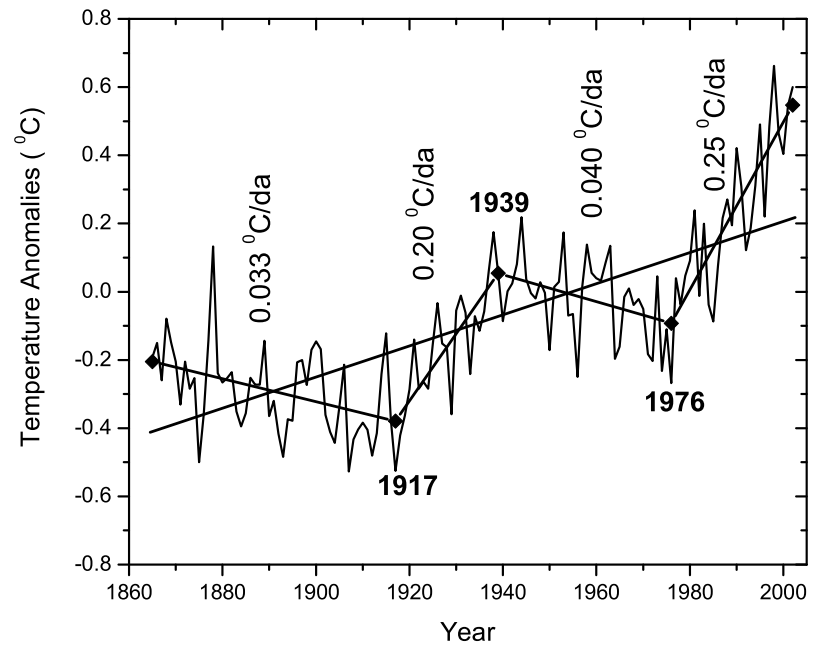

Fig. 3. Fitting of Northern Hemisphere temperature anomaly with continuous line segments. The minimum amount of trend change at breakpoints is $0.2^{\circ} \mathrm{C} / \mathrm{dec}$ ade, and 20 years is the minimum allowed interval between breakpoints ( 5 years for end segments).

there is still an ample debate about the statistical support for those conclusions. The reason is apparent in Fig. 1, considering the fact that the mean decadal change of the NAO index is quite small in view of its large interannual variability. In Sect. 5 it will be shown that the methodology here proposed can recover partial trends from synthetic series, defined in its simplest sense as monotonous changes over some time interval, in the presence of significant levels of noise. It will also be shown that the critical point in the performance of the method is the location of the breakpoints and that once these are known, the partial trend values are not much affected by the amplitude of noise.

The breakpoints of NAO presented in Figs. 1 and 2 differ slightly from those presented by Tomé and Miranda (2004) because in that study end effects were not considered. In that study, a change of sign was always imposed at breakpoints but that didn't affect the solution in this particular case.

The simultaneous imposition of limits for the segment length and for the change in trend at breakpoints seems a sensible way of constraining the fitting. In the case shown, the imposed minimum segment size allowed a maximum of 7 breakpoints, but the condition of minimum trend change reduced it to 4 breakpoints.

\subsection{The Northern Hemisphere temperature record}

The Climate Research Unit (CRU), University of East Anglia, produces and frequently updates a database of the global temperature that has been the basis of many global warming studies. The data is freely available at http://www.cru.uea. ac.uk/cru/data/ and is described in Jones et al. (1999) and Jones and Moberg (2003). Figure 3 shows the mean Northern hemisphere temperature between 1865 and 2002, together with the linear fit and the fit with continuous line segments with a minimum trend change at breakpoints of $0.2^{\circ} \mathrm{C} / \mathrm{decade}$ and a minimum of 20 years between breakpoints, except at the end segments where a minimum length of 5 years is allowed.

The long term change of $\mathrm{NH}$ temperature is estimated as $0.63^{\circ} \mathrm{C}\left(0.046^{\circ} \mathrm{C} /\right.$ decade $)$, whereas the corresponding estimate by the proposed fitting method is slight higher at $0.75^{\circ} \mathrm{C}$ $\left(0.054^{\circ} \mathrm{C} /\right.$ decade $)$. More importantly, the non-linear fit captures important information on the interdecadal evolution of surface temperature, detecting two cooling periods (18651917 and 1939-1976) and two warming periods (1917-1939 and 1976-2002), with a good visual fit to the data. A remarkable result, in the context of the climate change debate, is the high trend value in the most recent warming period $\left(0.25^{\circ} \mathrm{C} / \mathrm{decade}\right)$. This results are comparable with those obtained by Karl et al. (2000) for the global world temperature.

Once again, one may conclude that the condition on the minimum trend change is an important constraint on the number of breakpoints. For this time series the chosen window size allows for 7 breakpoints but the conditions of a minimum trend change of $0.2^{\circ} \mathrm{C} /$ decade reduce that number down to 3 breakpoints. In this case, the solution obtained for three breakpoints without additional conditions for the minimum trend change is not the same as presented in Fig. 3, although it is quite close. The unconstrained set of 4 line segments that best fits the time series has breakpoints at 1912, 1943 and 1975, with partial trends of $-0.043,0.15,-0.053$ and $0.24^{\circ} \mathrm{C} /$ decade, respectively. This solution was not selected in the first place because both at 1912 and 1943 the changes in trend are smaller than $0.2^{\circ} \mathrm{C} /$ decade. However, the two solutions coincide if one chooses a minimum trend change of $0.15^{\circ} \mathrm{C} /$ decade at the breakpoints. In these cases, slightly different values of breakpoint positions can lead to a slightly better fit, and the user has to choose the preferred solution.

\subsection{A case with a large number of data points: Hemispheric} multi-proxy temperature reconstructions

The proposed methodology requires a huge computation time whenever the time series contains a large number of data points. In those cases, it is possible to make the problem treatable by carefully subdividing the time series into a small number of overlapping sub intervals in order to find the breakpoint positions in the time series. After finding the breakpoint positions, one only needs to solve the over determined system linear equations (3). It is important that the beginning of a sub interval coincides with a breakpoint found in the previous subinterval. Preferably, the breakpoint chosen for interval boundary should be sufficiently away from the boundaries and other breakpoints by a distance larger than the minimum allowed interval.

There are many large geophysical time series that one could choose as an example to illustrate the above mentioned workaround. We will use the historical reconstruction of the Northern hemisphere temperature by Mann and Jones (2003). 


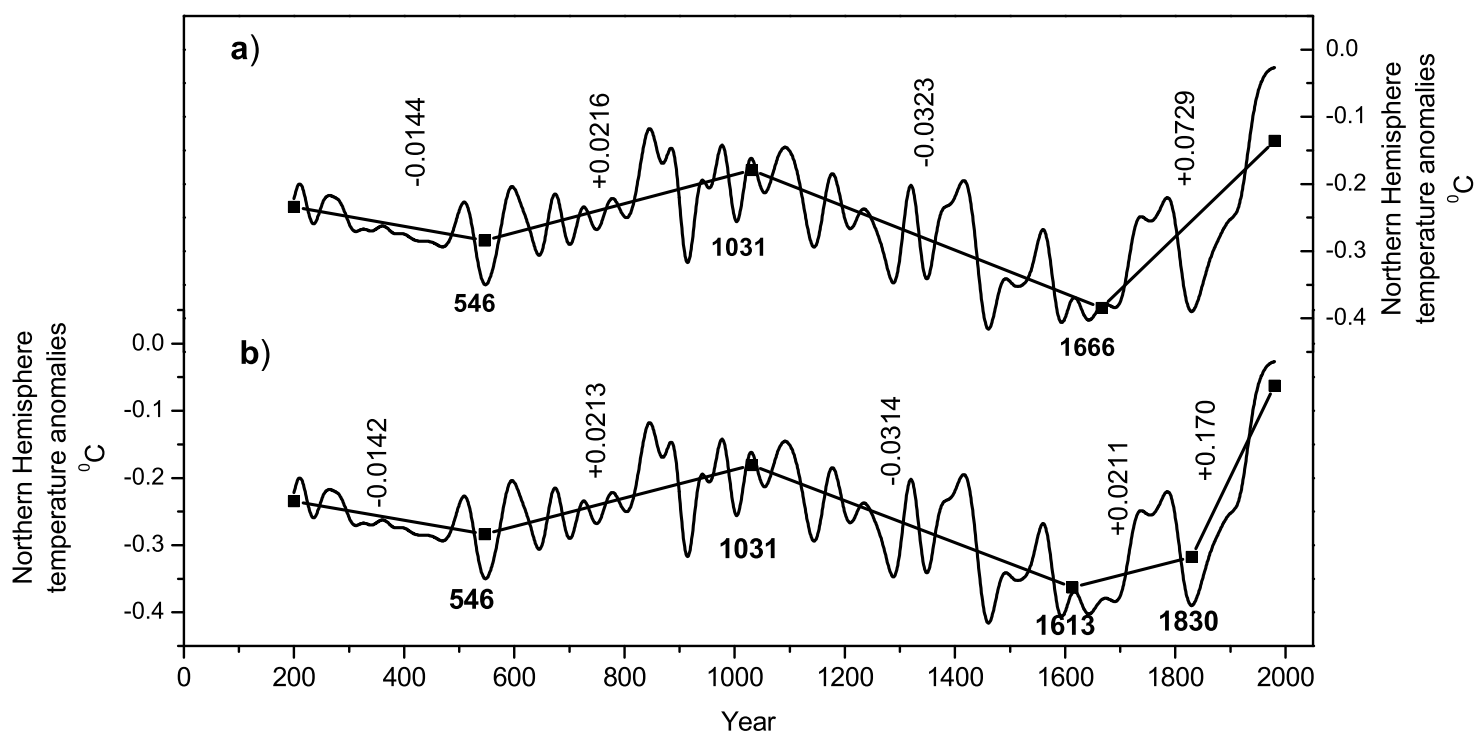

Fig. 4. Fitting of the Northern Hemispheric temperature anomalies, based on 1961-1990 instrumental reference period, with continuous line segments. (a) 200 years minimum distance between breakpoints, and change of trend sign at breakpoints. (b) As (a), but the last interval was allowed to have a minimum length of 150 years and the change in trend at breakpoints is not less than $300 \%$.

Figure $4 \mathrm{a}$ presents a fit to a series of 1781 years of hemispheric multi-proxy temperature evaluated by Mann and Jones (2003), freely available at ftp://ftp.ngdc.noaa.gov/ paleo. In this case, the best fit was obtained, for a condition of change in the trend sign at breakpoints and a minimum of 200 years between breakpoints, with breakpoints at the years of 546, 1031 and $1666 \mathrm{AD}$. The preprocessing of the time series in 3 sub-intervals led to a reduction of computing time by a factor of $10^{6}$ (from almost half a century, virtually impracticable in a single computer, to less than half and hour in a Pentium IV PC).

The best fit of the proxy temperature data estimates a decrease of the mean temperature in the first 346 years (200 till $546 \mathrm{ac}$.) at a rate of $-0.014^{\circ} \mathrm{C} /$ century, followed by a temperature increase at a rate of $0.022^{\circ} \mathrm{C} /$ century until 1031, then a very long period, more than 6 centuries, of continuous temperature decrease at a rate of $-0.032^{\circ} \mathrm{C} /$ century. The large rate of warming after $1666,0.073^{\circ} \mathrm{C} /$ century, has already been able to compensate the previous 6 centuries of cooling and the last segment attains temperature values above the local maximum at 1031 , at the centre of the warm medieval period AD 800-1400 (Mann and Jones, 2003).

In spite of the relatively good overall fit presented in Fig. 4a, the large single oscillation present in the end of the proxy temperature time series suggests that one may be underestimating the latter partial trend, due to an end effect related with the minimum of 200 years of trend duration. Figure $4 \mathrm{~b}$ presents an alternative fit of the same data of Fig. $4 \mathrm{a}$ with the following modification of the optimization conditions: sign change or a minimum relative variation of $300 \%$ in the trend value is imposed at the breakpoints, and a minimum length of 150 years is allowed for the last line segment. The main difference from the result in Fig. $4 \mathrm{a}$ is a last trend beginning at 1830 at a faster warming rate of $0.17^{\circ} \mathrm{C} /$ century, much higher than elsewhere in the series, but still below estimates based on thermometer data in the last century.

\section{A spatial aggregation technique}

The proposed methodology is clearly a univariate time series analysis technique. However, it defines for a given time series a new set of parameters, the breakpoints, that can be used to perform spatial aggregation of a time varying field. The idea was proposed by Miranda and Tomé (2005) and applied to the NASA/GISS surface temperature dataset (Hansen et al., 1999), leading to the conclusion that the spatial distribution of the temperature breakpoints in the last 50 years is a coherent large scale field, that may be relevant for the understanding of the slow evolution of the climate system in that period.

To illustrate the potential use of the method as a spatial analysis tool we will apply it to a different climate database, namely the NCEP/NCAR $2 \mathrm{~m}$ reanalysis temperature (Kalnay et al., 1996), available from 1950 onwards and obtained through the assimilation of meteorological observations by a state of the art numerical weather prediction model. Reanalysis data has been used for climate change studies (Kalnay and Cai, 2003) but is generally discarded for this application because of time dependent biases in the data that is used by reanalysis, namely in what concerns satellite data (Basist and Chelliah, 1997; Hurrell and Trenberth, 1998; Santer et al., 1999, 2000; Stendel et al., 2000). However, it is ideally suited for our current purpose as it has a global coverage, without the missing data that often hinders the use of direct observations. 


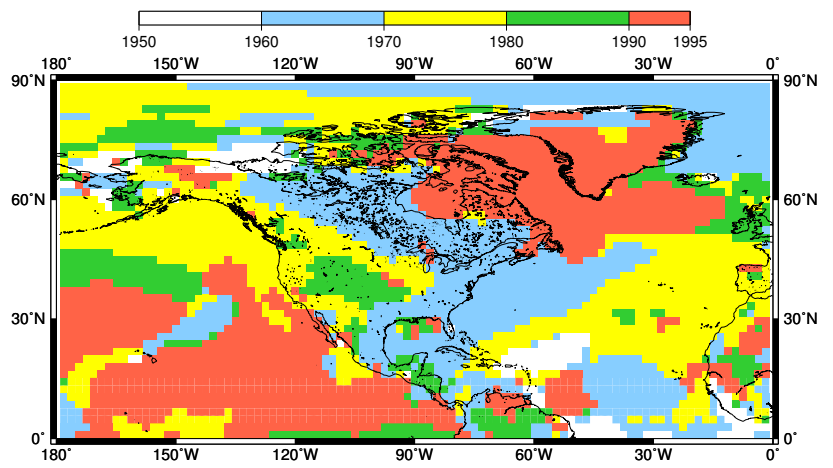

Fig. 5. Year of change in sign of local temperature tendency. Blank in regions with no breakpoint.

Considering the evolution of mean world temperature one would expect one single breakpoint in the period 1950-2002 with a cooling period followed by warming. For mean world temperature that breakpoint should be around 1975 as shown by (Karl et al., 2000). However, a point by point analysis of the reanalysis time series shows that the breakpoint year varies from place to place in a rather consistent way. Figure 5 shows the breakpoint distribution in the North American region, from a best fit of the NCEP/NCAR $2 \mathrm{~m}$ temperature reanalysis field where only two line segments are allowed and a change in trend sign is imposed at the breakpoint. White regions in the figure are places of monotonous temperature change, mostly sustained warming, where a breakpoint with a trend sign change cannot be defined. Ten years was the minimum allowed length for each of the sub intervals.

The North American region is where we find a larger discrepancy between estimates of long term change in temperature given by a linear trend without breakpoint and by the linear trend with one breakpoint. In this region, the linear trend gives, in some places, strong negative values that are not supported by the integrated segments, as the linear fit in the temperature time series is obviously inadequate.

Figure 6 presents the net change in temperature in the North American region, estimated by linear regression and by the integration of the two fitted segments. While the simple linear fit leads to large net cooling, especially over Greenland and Baffin Bay and in a large sector of the USA territory, the broken line approach suggests moderate net cooling in most places, with recent warming compensating for previous cooling in many regions, namely in half of Greenland.

An important feature of the breakpoint map shown in Fig. 5 is the fact that the observed breakpoints span the entire four decades range in a spatially consistent way, as one could expected by the spatial correlation of the reanalysis fields. However, Miranda and Tomé (2005) obtained a similar pattern with GISS data and suggested that this is a signature of a slow evolution of the climate heat transfer engine in the region, associated with the NAO evolution in the same period. The spatial coherency of the breakpoint distribution, with neighboring grid points experiencing similar tempera-
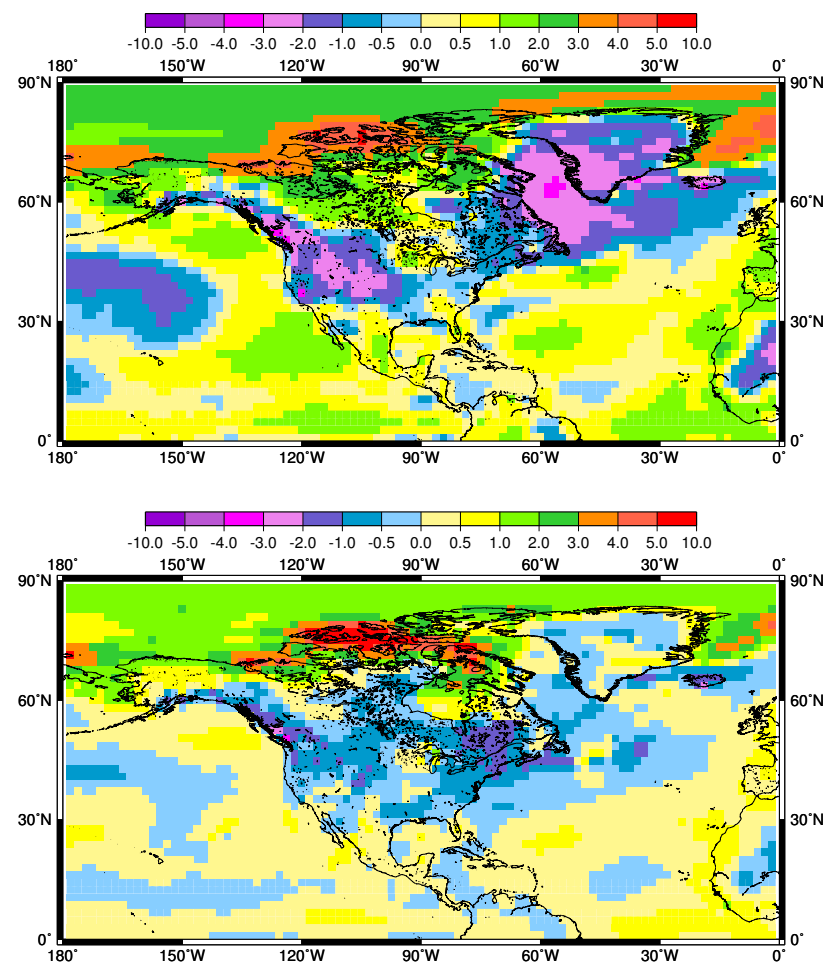

Fig. 6. Temperature variation of the mean surface temperature, in ${ }^{\circ} \mathrm{C}$ : upper figure linear variation, lower figure two continuous line best fit.

ture changes, also suggests the spatial aggregation of regions with similar breakpoint locations, as done by Miranda and Tomé (2005). Other time varying geophysical fields may be analyzed with the same methodology.

\section{Statistical significance}

An important and unavoidable issue is the statistical significance of the breakpoints and/or the partial trends. At first glance, one may be tempted to test the individual partial trends for significance using some of the many techniques that have been proposed by several authors in the last decades (e.g. Gordon, 1991; Bloomfield, 1992; Bloomfield and Nychka, 1992; Visser and Molenaar, 1995; Zheng et al., 1997) and consider as statistical significant those results above a given threshold of the confidence interval. However, in spite of the huge effort put in the analysis of the significance of the simple linear trend model, a simple and objective method has not yet obtained general consensus, and the different models lead to different conclusions, not just in the value and statistical significance of a trend, but also in the validity of the trend model itself.

Karl et al. (2000) applied standard significance tests to discuss the trend computed for the last segment in a broken-line fitting of the mean world temperature. It is not possible to extend that approach to the full problem, which includes the computation of the number of breakpoints, its location and 


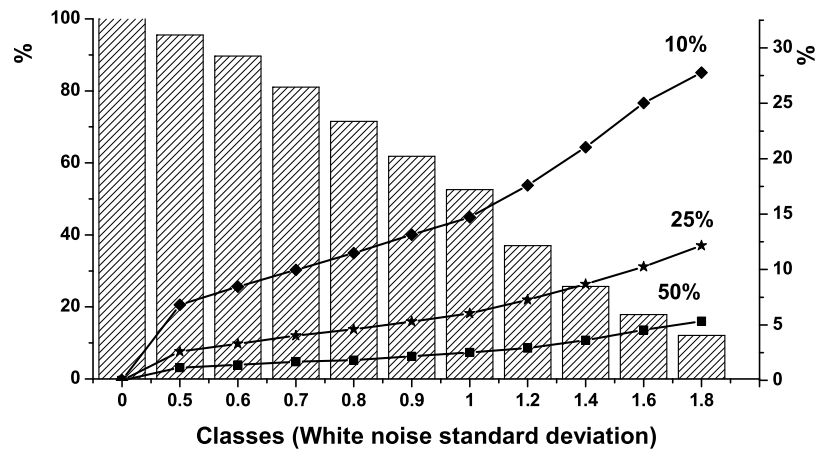

Fig. 7. Performance of the broken-line fitting as a function of the error level. Bars (left axis): Percentage of breakpoints recovered without error. Lines (right axis): Frequency of errors in the partial trends above different thresholds, for the cases where the breakpoint inversion was successful. The level of noise (low axis) is given by the standard deviation of the white noise.

the evaluation of partial trends, with a condition of continuity of the fitting function at the breakpoints. If, as in the case of DFA (Peng et al., 1994), one specifies the subinterval range and then applies unconstrained linear fit, without a condition of continuity at the subinterval boundaries, one could test for the individual significance of each of the individual partial trends. Yet, in that case, one should be aware of the biased conclusions on significance due to the sub interval chosen, because in that particular case the warnings about the significance of the trend value formulated by Percival and Rothrock (2005) are especially pertinent and should be taken into account.

While a general test is difficult to obtain, one can easily check the ability of the proposed method to cope with the difficulties of real data, by the analysis of a large number of simulations with synthetic time series. For that purpose we have computed 10000 non-dimensional time series, of 100 data points each, made of random continuous line segments with a minimum length of 15 data points and a minimum change in trend of $0.1 /$ datapoint at breakpoints. The trend values were in the range $[0,1] /$ datapoint. With these conditions the random series could have at most 6 line segments (5 breakpoints). Situations with only one line segment (zero breakpoints) were also allowed. As expected, the method was able to recover all the breakpoints (or its absence) and corresponding trend values from this series. In the next step we added to these random series white noise of varying amplitude.

Figure 7 shows the results of the inversion of the 10000 random series, for different values of the noise level. When there is no noise, the inversion works at $100 \%$, recovering all breakpoints at the right location and computing all partial trends at round-off error. In the presence of significant noise (with standard deviations from 0.5 to 1.8 in non-dimensional units) the frequency of misfits increases steadily with the noise level, as shown in the figure. However, for the fraction of cases where the breakpoints were found within 2 dat-

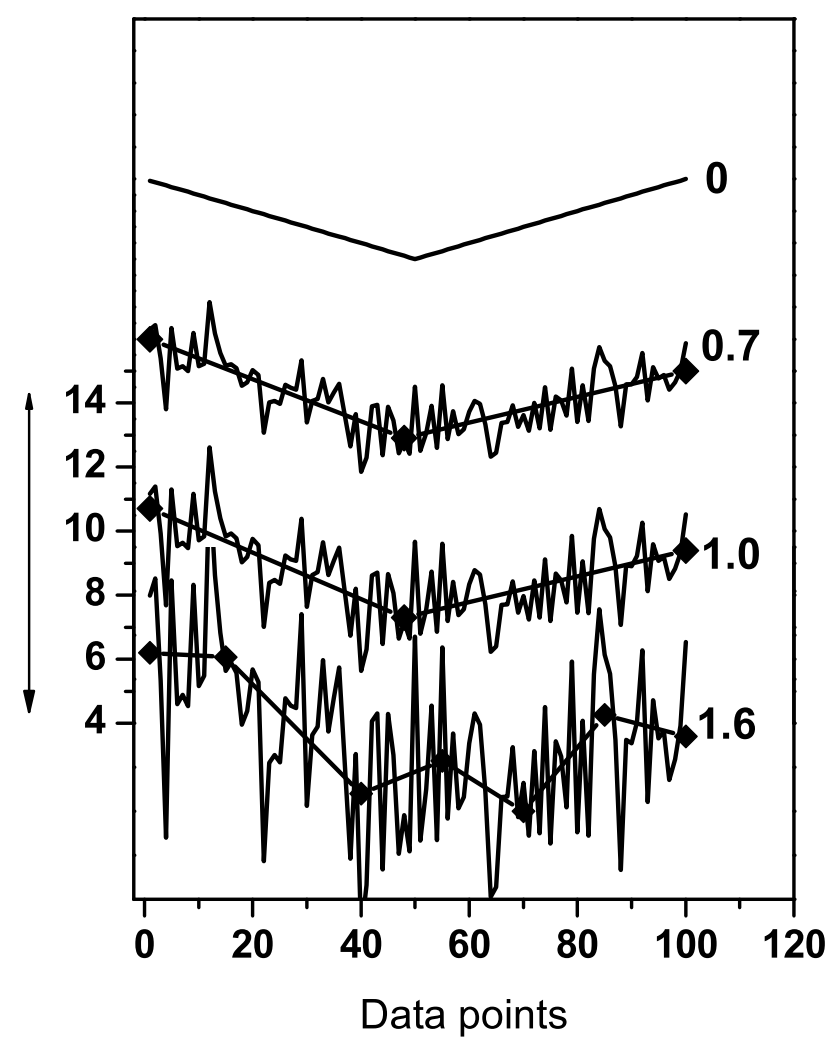

Fig. 8. Results of the inversion of one of the series used in Fig. 7 for different noise levels.

apoints of their right location, which are considered to have been well located, the maximum relative error in the partial trends remains within reasonable bounds, even when the error level is such that the fraction of misfits is above $50 \%$. This means that if the breakpoint location is known the partial trends are not too sensitive to the noise. On the other hand, most of the cases that present larger relative errors in the partial trends happen in the last segment, because it is the least constrained.

Figure 8 shows an example of the inversion of a series with 1 breakpoint, taken from the set used in Fig. 7. The top line corresponds to the series without any added noise, which is perfectly inverted. The series with 0.7 and 1.0 noise also lead to successful inversions, in spite of the large random oscillations present in the series. However, when the noise standard deviation attains 1.6 , the method proposes the maximum allowed number of breakpoints. An eye inspection of this result suggests, though, that the proposed fitting is not at all unrealistic.

The random series considered in Fig. 7 were rather difficult to invert due to the small relative size of its shorter line segments and the small changes of trend allowed at each breakpoint. If either of these parameters is increased, the method is able to cope with much larger noise levels. Figure 9 shows the results of 3 other sets of 10000 random series, similar to those used in Fig. 7 but with at least 20, 25 and 30 datapoints per segment, respectively. As the mini- 
(a)

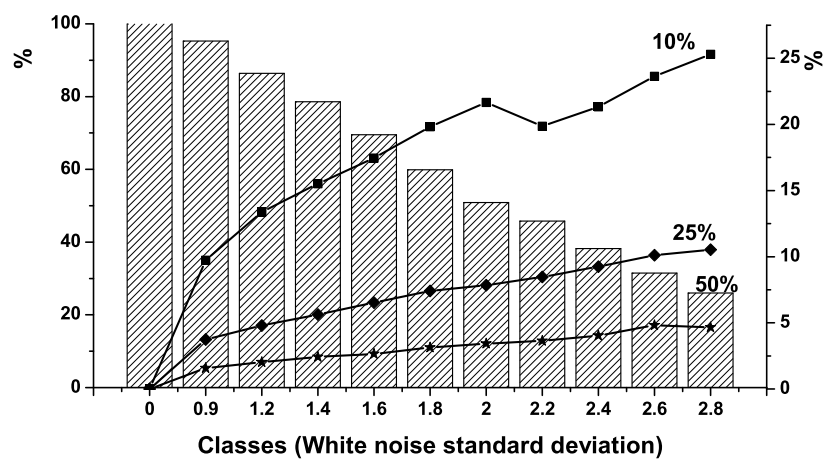

(b)

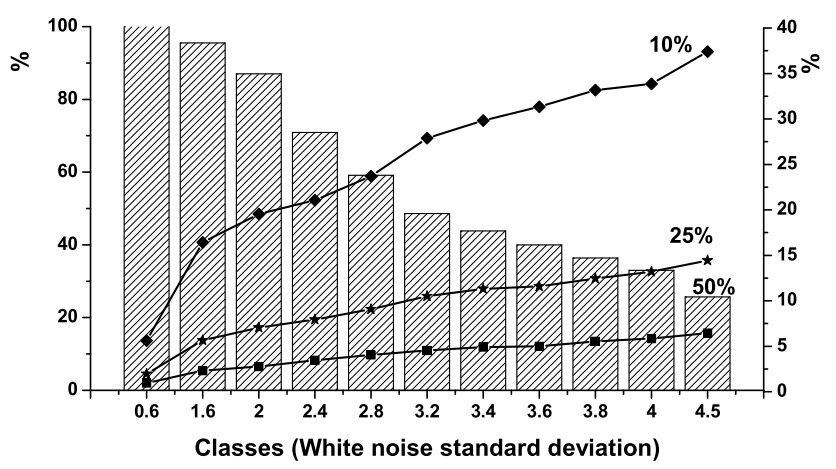

(c)

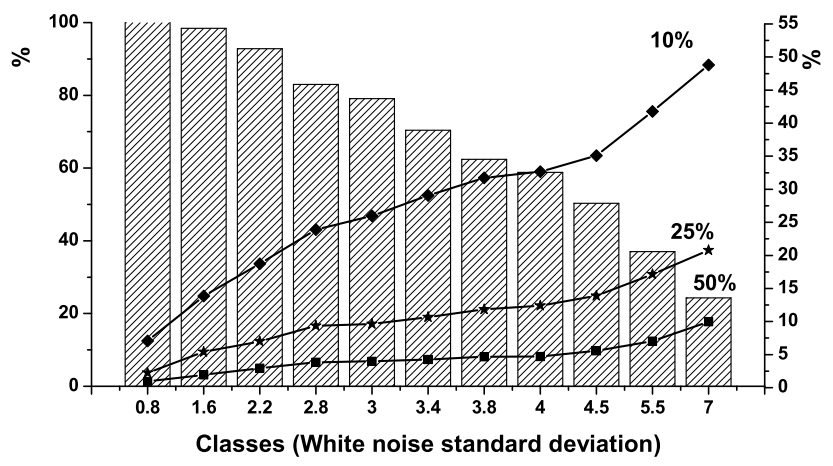

Fig. 9. Same as Fig. 7 but the 10000 random series were generated with a minimum of 0.1 trend change at breakpoint and (a) 20 datapoints as minimum length (b) 25 datapoints as minimum length (c) 30 datapoints as minimum length.

mum size of the segments increases, the performance of the inversion improves substantially. If one looks at the noise level corresponding to $50 \%$ of successful breakpoint locations, it goes from about 1.1 (for a minimum size of 15 datapoints per segment) to about 5 (for 30 datapoints). Again, one finds that, even at very large noise levels, once the breakpoints are rightly located most inversions lead to small errors in the partial trends. It is interesting to note, in a visual com- (a)

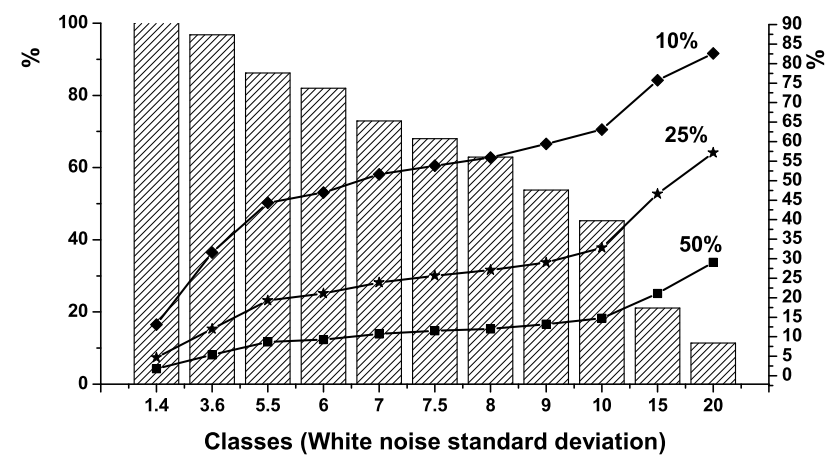

(b)

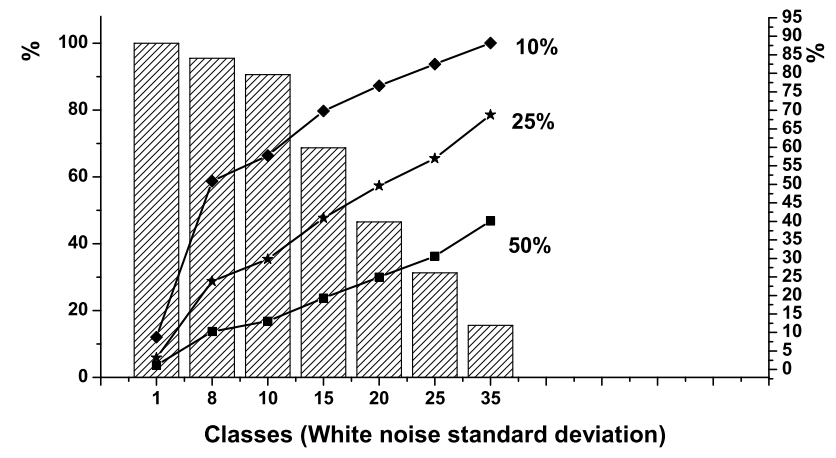

Fig. 10. Same as Fig. 9b but the 10000 series random were generated in the top panel with a minimum of 0.3 trend change at breakpoint and in the bottom panel with a minimum of 0.6 trend change at breakpoints.

parison between Fig. 7 and the 3 panels of Fig. 9, that there is a strong similarity between the different curves, suggesting that the allowed noise levels scale with the size of the minimum segment size.

On a second set of sensitivity experiments one will now look to the effect of trend variations at the breakpoint. Figure 10 shows results of the inversion for 2 sets of 10000 random series where the minimum trend change at each breakpoint has been set to 0.3 and 0.6 /datapoint, respectively, with a minimum segment size of 25 datapoints. As expected, an increase in the trend differences between segments improves the performance of the method, allowing for increased noise levels by a factor that, again, scales with the change in trends.

The previous sensitivity experiments indicate that the method behaves in a sensible way as a function of its constraints. While we are not in a position to propose a general objective procedure to analyze the statistical significance of the breakpoints, one can perform simulations similar to those shown here to test specific geophysical series by comparison with large sets of random series.

The results presented in Figs. 7, 9 and 10 refer to the cases where all breakpoints were found. On a breakpoint by breakpoint basis results are substantially better, event at large noise 
levels. However, it is easy to understand that once one breakpoint is lost in the inversion process, it leads to large errors in the adjacent partial trends.

To conclude, one may say that, in spite of recent advances in trend analysis, the statistical and physical significance of trends is still an open discussion. The methodology here proposed adds another problem to that discussion: how to test the significance of breakpoints in the fitting of a series by continuous line segments? The approach followed in this section tried to show the feasibility of the algorithm proposed and its relative robustness in the presence of significant noise. However a more general significance test, able to deal with other stochastic data models besides white noise, would certainly contribute to the usefulness of the methodology.

\section{Conclusions}

Fitting continuous line segments to time series provides a new and alternative way of analyzing non-monotonic and non-periodic time series. The method is able to identify sensible changes in the low frequency evolution of the time series and is therefore far richer than simple linear trend analysis. The method is also much simpler and easier to interpret than most non-linear function fitting. In many cases it leads to results that are similar to subjective curve analysis, while keeping a least squares based approach constrained by a small number of sensible conditions. Because the method gives a continuous fit of the data it always provides a global analysis. However, if the conditions are carefully chosen, it adjusts itself to sharp changes in the series, highlighting important episodes in the series history that may be more important than its overall trend. One question remains, unfortunately, without an appropriated answer: how to rightfully choose the conditions?

The output of the proposed method includes, together with an estimate of the slow change in the data, a set of breakpoints that may be used for different purposes in the analysis. The breakpoints constitute a small set of parameters that characterize the slow evolution of the geophysical system being analyzed. It was shown that they may help in the analysis of time varying and spatially distributed geophysical series.

The method implementation was based on solving, several times, in the least squares sense, $L_{2}$-norm, a linear over determined equation system (Eq. 3). Nevertheless, this modular approach allows one to choose other measures of the error, as for instance the absolute residual sum, $L_{1}$-norm, the absolute maximum departure, $L_{\infty}$-norm, or even other more complex departure measures.

The results of the method depend mostly on the minimum allowed distance between breakpoints, and also on the minimum trend change. The option on a minimum trend change gives an objective meaning to the breakpoints and is the kernel for determining the number of line segments to fit. Sensitivity tests with a large number of series, made of line segments with random sizes and slopes and white noise of varying amplitude, indicated that the methods is able to recover the right position of breakpoints and the values of the partial trends even for relatively large values of the noise. Those results also showed that the location of breakpoints is the critical issue in the performance of the method.

As happens with other methods dealing with non-periodic and non-monotonic time series, the end effects are a problematic issue. The option to minimize these effects was to allow, at the beginning and at the end of the record, shorter segment lengths. Once again, if the first or the last breakpoint happens near the minimum allowed position the result should be looked upon with some suspicion.

The method was implemented in a FORTRAN 90 program. The source code can be downloaded at http://www. dfisica.ubi.pt/ artome/linearstep.html or can be requested by mail from AT.

Acknowledgements. This study was developed at CGUL, partially supported by FCT through Grant POCTI/CTE-ATM/46573/2002, co-financed by the EU under program FEDER. A. R. Tomé benefited from a sabbatical leave from the Universidade da Beira Interior. Suggestions made by an anonymous referee have contributed to improve the paper.

Edited by: M. Thiel

Reviewed by: two referees

\section{References}

Basist, A. N. and Chelliah, M.: Comparison of tropospheric temperatures derived from the NCEP/NCAR reanalysis, NCEP operational analysis and the Microwave Sounding Unit, Bull. Am. Met. Soc., 78, 1431-1447, 1997.

Bloomfield, P.: Trend in global temperature, Climate change, 21, $1-16,1992$.

Bloomfield, P. and Nychka, D. W.: Climate spectra and detecting climate change, Climate Change, 21, 275-287, 1992.

Bouaricha, A. and Schnabel, R. B.: Equations and Nonlinear Leastsquares Problems Using Tensor Methods, Transactions on Mathematical Software, 232, 21, 174-195, 1997.

Gordon, A. H.: Global warming as a manifestation of a random walk, J. Climate, 4, 589-597, 1991.

Hansen, J., Ruedy, R., Glasdoe, J., and Sato, M.: GISS analysis of surface temperature change, J. Geophys. Res., 104, 30997 $31022,1999$.

Hansen, J., Ruedy, R., Sato, M., Imhoff, M., Lawrence, W., Easterling, D., Peterson, T., and Karl, T.: A closer look at United States and global surface temperature change, J. Geophys. Res., 106, 23 947-23 963, 2001

Hurrell, J. W.: Decadal trends in the North Atlantic Oscillation: regional temperatures and precipitation, Science, 269, 676-679, 1995.

Hurrell, J. W. and Trenberth, K. E.: Difficulties in obtaining reliable temperature trends: reconciling the surface and satellite $\mathrm{Mi}-$ crowave Sounding Unit records, J. Climate, 11, 945-967, 1998.

Jones, P. D., Jonsson, T., and Wheeler, D.: Extension to the North Atlantic Oscillation Using Early Instrumental Pressure Observations From Gibraltar and South-West Iceland, Int. J. Clim., 17, 1433-1450, 1997. 
Jones, P. D., New, M., Parker, D. E., Martin, S., and Rigor, I. G.: Surface air temperature and its variations over the last 150 years, Reviews of Geophysics, 37, 173-199, 1999.

Jones, P. D. and Moberg, A.: Hemispheric and large-scale surface air temperature variations: An extensive revision and an update to 2001, J. Climate, 16, 206-223, 2003.

Karl, T. R., Knight, R. W., and Baker, B.: The record breaking global temperatures of 1997 and 1998: Evidence for an increase in the rate of global warming?, Geophys. Res. Lett., 27, 719-722, 2000.

Kalnay, E. and Cai, M.: Impact of urbanization and land use change on climate, Nature, 423, 528-531, 2003.

Kalnay, E., Kanamitsu, M., Kistler, R., Collins, W., Deaven, D., Gandin, L., Iredell, M., Saha, S., White, G., Woollen, J., Zhu, Y., Leetmaa, A., Reynolds, B., Chelliah, M., Ebisuzaki, W., Higgins, W., Janowiak, J., Mo, K. C., Ropelewski, C., Wang, J., Jenne, R., Joseph, D.: The NCEP/NCAR 40-Year Reanalysis Project, Bulletin of the American Meteorological Society: 77, 437-472, 1996.

Mann, M. E. and Jones, P. D.: Global Surface Temperatures over the Past Two Millennia, Geophys. Res. Lett., 30, 15, 1820, doi:10.1029/2003GL017814, 2003.

Mann, M. E.: On smoothing potentially climate time series, Geophys. Res. Lett., 31, L07214, doi:10.1029/2004GL019569, 2004.

Miranda, P. M. A. and Tomé, A. R.: Large scale spatial structure of observed temperature trends, in Proc. 16th Conference on Climate Variability and Change, American Meteorological Society, 2005 .

Ostermeier, G. M. and Wallace, J. M.: Trends in the North Atlantic Oscillation -Northern Hemisphere Annular Mode during the Twentieth Century, J. Climate, 16, 336-341, 2003.

Peng, C. K., Buldyrev, S. V., Havlin, S., Simons, M., Stanley, H. E., and Goldberger, A. L.: Mosaic Organization of DNA Sequences, Phys. Rev. E, 49, 1685-1689, 1994.

Percival, D. B. and Rothrock, D. A.: Eyeballing, Trends in Climate Time Series: A Cautionary Note, J. Climate, 18, 886-891, 2005.
Rayner N. A., Parker, D. E., Horton, E. B., Folland, C. K., Alexander, L. V., Rowell, D. P., Kent, E. C., and Kaplan, A.: Global analyses of sea surface temperature, sea ice, and night marine air temperature since the late nineteenth century, J. Geophys. Res., 108, D14, 4407, doi:10.1029/2002JD002670, 2003.

Santer, B. D., Hnilo, J. J., Wrigley, T. M. L., Boyle, J. S., Doutriaux, C., Fiorino, M., Parker, D. E., and Taylor, K. E.: Uncertainties in observational based estimates of temperature change in the free atmosphere, J. Geophys. Res., 104, 6305-6333, 1999.

Santer, B. D., Wigley, T. M. L., Boyle, J. S., Gaffen, D. J., Hnilo, J. J., Nychka, D., Parker, D. E., and Taylor, K. E.: Statistical significance of trend differences in layer-average temperature time series, J. Geophys. Res., 105, 7337-7356, 2000.

Soon, W. W. H., Legates, D. R., and Baliunas, S. L.: Estimation and representation of long-term ( $>40$ year) trends of NorthernHemisphere gridded surface temperature: A note of caution, Geophys. Res. Lett., 31, L03209, doi:10.1029/2003GL019141, 2004.

Stendel, M., Christy, J. R., and Bengtsson, L.: Assessing levels of uncertainty in recent temperature time series, Clim. Dyn., 16, 8 , 587-601, 2000.

Tomé, A. R. and Miranda, P. M. A.: Piecewise linear fitting and trend changing points of climate parameters, Geophys. Res. Lett., 31, L02207, doi:10.1029/2003G019100, 2004.

Vautard, R. and Ghil, M.: Singular spectrum analysis in non-linear dynamics with applications to paleoclimatic time series, Physica D, 35, 395-424, 1989.

Vautard, R., Yiou, P., and Ghil, M.: Singular spectrum analysis: A toolkit for short, noisy chaotic signal, Physica D, 58, 95-126, 1992.

Visser, H. and Molenaar, J.: Trend estimation and regression analysis in climatological time series: An application of structural time series models and the Kalman filter, J. Climate, 8, 969-979,1995.

Zheng, X., Basher, R. E., and Thompson, C. S.: Trend detection in regional-mean temperature series: Maximum, minimum, mean, diurnal range, and SST, J. Climate, 10, 317-326, 1997. 